\title{
A Novel Pharmacogenetic Model for Highly Efficient Ablation of Oligodendrocyte Progenitor Cells in the Adult Mouse CNS
}

Yao Lulu Xing ${ }^{1}$, Bernard H.A. Chuang ${ }^{1}$, Jasmine Poh $^{1}$, Kaveh Moradi ${ }^{1,2}$, Stanislaw Mitew $^{1}$, Trevor J. Kilpatrick ${ }^{2}$, Yasuyuki Osanai ${ }^{1,3}$, Tobias D. Merson* ${ }^{1}$

${ }^{1}$ Australian Regenerative Medicine Institute, Monash University, Clayton, Victoria, Australia; ${ }^{2}$ Florey Institute of Neuroscience and Mental Health, Parkville, Victoria, Australia; ${ }^{3}$ Current address: Division of Histology and Cell Biology, Department of Anatomy, Jichi Medical University, Tochigi, Japan.

*Correspondence should be addressed to T.D.M. (tobias.merson@monash.edu).

\section{Acknowledgements:}

This work was supported by funding from the Australian Research Council Special Research Initiative - Stem Cells Australia (T.D.M., T.J.K.). Y.L.X received support from The CASS Foundation. T.D.M received support from an Australian Research Council Future Fellowship (FT150100207) and Metal Manufactures Ltd. We would like to thank Professor William Richardson (University College London, UK) for generously providing the Pdgfra-CreER $R^{T 2}$ and Sox10-lox-GFP-STOP-lox-DTA transgenic mouse lines. The authors would also like to acknowledge use of facilities within the Monash Micro Imaging Platform, Monash Histology Platform and Micromon (Monash University).

\section{Author Contributions:}

Y.L.X., T.J.K. and T.D.M. designed research. Y.L.X., Y.O., B.H.A.C., J.P., K.M., S.M. and T.D.M. performed research. Y.L.X. and T.D.M. wrote the paper. 


\title{
Competing Interests statement:
}

The authors declare no competing financial interests.

\begin{abstract}
Approaches to investigate adult oligodendrocyte progenitor cells (OPCs) by targeted cell ablation in the rodent central nervous system have been limited by methodological challenges resulting in only partial and transient OPC depletion. We have developed a novel pharmacogenetic model of conditional OPC ablation, resulting in the elimination of $99.7 \%$ of all OPCs throughout the brain. By combining recombinase-based transgenic and viral strategies for targeting of OPCs and ventricular-subventricular zone (V-SVZ)-derived neural precursor cells (NPCs), we found that new PDGFRA-expressing cells born in the V-SVZ repopulated the OPCdeficient brain starting 12 days after OPC ablation. Our data reveal that OPC depletion induces V-SVZ-derived NPCs to generate vast numbers of $\mathrm{PDGFRA}^{+} / \mathrm{NG}^{+}$cells with the capacity to migrate and proliferate extensively throughout the dorsal anterior forebrain. Further application of this novel approach to ablate OPCs will advance knowledge of the function of both OPCs and oligodendrogenic NPCs in health and disease.
\end{abstract}




\section{Introduction}

Oligodendrocyte progenitor cells (OPCs), also known as NG2-glia, are the principal mitotic cell type in the adult mammalian central nervous system (CNS). OPCs are known primarily for their capacity to generate myelin-forming oligodendrocytes (OLs) during postnatal development and adulthood ${ }^{1,2}$. Whilst OPCs are distributed fairly uniformly throughout the CNS, including in brain regions where relatively little myelination occurs, they exhibit a high degree of phenotypic and functional heterogeneity between brain regions ${ }^{3-5}$, which has raised the prospect that they could possess additional functions beyond oligodendrogenesis.

To investigate the function of OPCs within the adult CNS, a number of groups have developed different strategies to selectively ablate OPCs. However, these experimental approaches including X-irradiation, laser-mediated ablation, geneticallyinduced cell ablation, or the use of anti-mitotic drugs have their own specific caveats that have limited the extent to which it has been possible to achieve long-term depletion of OPCs in adult mice ${ }^{6-11}$. Existing approaches have enabled only partial and transient OPC ablation throughout the CNS, due to incomplete targeting of the OPC population and rapid repopulation by nearby unaffected OPCs. As a result, it has not yet been possible to explore the functional consequences of long-term OPC ablation.

Designing a genetic approach to both completely and selectively ablate OPCs in the adult mouse CNS requires careful consideration of the promoter(s) that will be used to target the OPC population. Parenchymal OPCs are defined by their expression of chondroitin sulfate proteoglycan 4/neuron-glial antigen $2(\mathrm{CSPG} 4 / \mathrm{NG} 2)^{12}$ and platelet derived growth factor receptor alpha (PDGFRA) ${ }^{13}$. Although the Cspg4 promoter has been used to control transgene expression for studies of parenchymal 
OPC fate, behavior and function ${ }^{1,9,14,15}$, NG2 is also expressed by pericytes ${ }^{8,10,16,17}$ and by some microglial cells after injury ${ }^{18}$. Similarly, PDGFRA is not an exclusive marker of OPCs; PDGFRA is also expressed by vascular and leptomeningeal cells (VLMCs, also known as perivascular fibroblasts) ${ }^{16,17}$ and by choroid plexus epithelial cells ${ }^{1,19,20}$. Therefore, the use of either the Cspg4 or Pdgfra promoter alone cannot direct the expression of a suicide gene exclusively to OPCs. In order to precisely target OPCs without affecting other neural cell types, we have generated a novel transgenic mouse model in which the expression of an inducible suicide gene is controlled by two different promoters, namely the Pdgfra and Sox10 promoters, whose overlapping transcriptional activity is restricted to OPCs in the CNS.

In addition to the specificity of genetic targeting, the method to conditionally ablate OPCs must also be highly efficient, targeting most, if not all, OPCs. High efficiency is critical for overcoming the proliferative response of non-ablated OPCs that follows incomplete OPC ablation and which has been demonstrated to result in swift regeneration of the OPC population ${ }^{7-9}$. Moreover, the method should be amenable to precise temporal control and have minimal effect on the overall health status of the animal. To date, no strategy for ablating the entire OPC population has been described that meets all of these requirements.

We have developed a pharmacogenetic approach to ablate OPCs in the adult mouse CNS that overcomes many of the limitations of previous approaches. The method involves the inducible and conditional expression of diphtheria toxin A (DTA) in adult OPCs followed by delivery of an anti-mitotic agent into the CNS to ablate dividing OPCs that escape genetic targeting. We demonstrate that this approach 
provides highly efficient and selective ablation of OPCs across the entire adult mouse brain, resulting in the loss of $99.7 \pm 0.2 \%$ of recombined OPCs.

Using this pharmacogenetic model of conditional OPC ablation also uncovered the remarkable compensatory regenerative response of the ventricularsubventricular zone (V-SVZ). Beginning 12 days after widespread OPC ablation, nestin-expressing neural precursor cells (NPCs) residing in the V-SVZ generated new $\mathrm{PDGFRA}^{+} \mathrm{NG}^{+}$cells whose morphology resembled those of OPCs. The newlygenerated PDGFRA ${ }^{+}$cells migrated extensively throughout the dorsal anterior forebrain, re-establishing the broad distribution of OPCs in this region of the brain. Collectively, our mouse model of conditional OPC ablation provides a valuable experimental tool for future studies aimed at better understanding the functions of OPCs and for further exploring the oligodendrogenic potential of NPCs residing in the V-SVZ. 


\section{Results}

\section{DTA-mediated ablation of OPCs induced rapid regeneration of the OPC}

\section{population}

To specifically ablate OPCs in the adult mouse CNS, we used an intersectional genetic approach to direct the inducible expression of a suicide gene in cells expressing both PDGFRA and SOX10. This was achieved by crossing two transgenic mouse lines, the Pdgfr $\alpha$-CreER $R^{T 2} \operatorname{line}^{13}$ and the Sox10-lox-GFP-STOP-lox-DTA ${ }^{21}$ (Sox10-DTA) line to enable DTA expression in adult OPCs upon delivery of tamoxifen (TAM) (Fig. 1a). Since the SOX10 transcription factor is expressed exclusively by cells of the oligodendroglial lineage in the $\mathrm{CNS}^{22,23}$, this ensures that DTA expression is restricted to OPCs and is not induced in either VLMCs or choroid plexus epithelial cells, both of which express PDGFRA but not SOX10.

TAM was administered to eight-week old Pdgfr $\alpha-C_{r e E R^{T 2+/+}}$ :Sox10-DTA ${ }^{+/-}$ mice (hereafter denoted Pdgfr $\alpha^{+}: D T A^{\dagger}$ ), as well as to Pdgfr $\alpha-C r e E R^{T 2+/+}:$ Sox $10-$ $D T A^{-/-}$littermates lacking the Sox10-DTA allele (denoted $P d g f r \alpha^{+}: D T A^{-}$), which served as non-ablated controls. Immunohistochemistry on the brains of non-ablated Pdgfr $\alpha^{+}: D T A^{-}$controls sacrificed 4 days after the final dose of TAM revealed abundant PDGFRA ${ }^{+}$OPCs throughout the brain, including the corpus callosum (CC) (Fig. 1b,c). By contrast, TAM administered $P d g f r \alpha^{+}: D T A^{+}$mice assessed at the same time-point had very few PDGFRA ${ }^{+}$OPCs in the corpus callosum (Fig. 1d), consistent with the notion that Cre-mediated induction of DTA resulted in OPC ablation. In these OPC-deficient mice, Sox 10-promoter driven GFP expression was restricted to SOX $10^{+} \mathrm{CC1}^{+}$cells (Supplementary Fig. 1a,b), indicating that Cre-mediated recombination of the Sox10-DTA allele targeted OPCs but not mature OLs. 
Although Pdgfr $\alpha^{+}: D T A^{+}$mice exhibited marked OPC depletion 4 days after TAM administration, OPC density in the corpus callosum returned to control levels by day 8 post TAM and increased further over the subsequent 2 days (Fig. 1e,f,g). Most OPCs present after 10 days were newly-generated, as demonstrated by the significant proportion of PDGFRA ${ }^{+}$cells that had incorporated 5-ethynyl-2'deoxyuridine (EdU) which was provided continuously in the drinking water after the last TAM administration (Supplementary Fig. 1c,d). Most OPCs present 4 days after TAM administration expressed GFP(Supplementary Fig. 1d), suggesting that the surviving OPCs were principally those in which the Sox10-DTA allele had not recombined. Some EdU ${ }^{+}$OPCs did not express GFP (Supplementary Fig. 1d), which most likely reflects low transcriptional activity of the Sox10 promoter that directs GFP expression. Supporting this idea, not all Sox $10^{+}$oligodendroglia in Pdgfr $\alpha^{+}$: $D T A^{+}$brains examined 4 days post TAM expressed GFP (Supplementary Fig. 1a). Overall, these data demonstrate that the vast majority of OPCs in Pdgfr $\alpha^{+}: D T A^{+}$mice were depleted after TAM administration. However, residual non-recombined OPCs exhibited a robust proliferative response to OPC ablation, resulting in restoration of OPCs to similar or higher density than non-ablated controls within 8-10 days after TAM administration.

\section{Intracisternal infusion of AraC after TAM administration prevented rapid OPC regeneration}

Given that incomplete OPC ablation triggered non-recombined OPCs that had escaped DTA-mediated apoptosis to proliferate and repopulate the CNS, we introduced a second intervention designed to kill these rapidly dividing OPCs. 
Specifically, after TAM administration, the antimitotic drug cytosine- $\beta$-D-

arabinofuranoside (AraC) was infused into the cisterna magna of $P d g f r \alpha^{+}: D T A^{+}$mice to deplete all residual proliferating OPCs. Osmotic minipumps were implanted on day 4 after TAM administration and removed on day 10 (Fig. 1h), to provide 6 days of continuous AraC infusion during the period of marked OPC proliferation. Vehicleonly controls received artificial cerebrospinal fluid (aCSF) without AraC. Mice were humanely sacrificed either immediately after removal of the osmotic minipump or 10 days later (Fig. 1h).

TAM-administered Pdgfr $\alpha^{+}: D T A^{+}$mice examined after 6 days of vehicle infusion, denoted as 0 days post pump removal (dppr), had numerous PDGFRA ${ }^{+}$ OPCs in the corpus callosum (Fig. 1i), similar in density to that observed in Pdgfr $\alpha^{+}$: $D T A^{+}$mice administered TAM alone and assessed 10 days later (Fig. 1f). By contrast, almost no PDGFRA ${ }^{+}$OPCs could be identified in the corpus callosum of TAMadministered $P d g f r \alpha^{+}: D T A^{+}$mice that were sacrificed immediately after AraC infusion (Fig. 1k). OPC depletion was sustained for at least 10 days after removal of

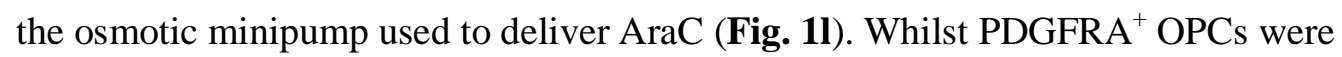
almost completely absent in TAM + AraC administered $P d g f r \alpha^{+}: D T A^{+}$mice, vascular-associated PDGFRA ${ }^{+} \mathrm{GFP}^{-}$cells surrounding PECAM-1 ${ }^{+}$endothelial cells in the brain parenchyma remained intact (Fig. 1m). These vascular-associated $\mathrm{PDGFRA}^{+} \mathrm{GFP}^{-}$cells in OPC-ablated mice did not exhibit typical ramified OPC morphology. We identified these vascular-associated PDGFRA ${ }^{+}$cells as laminin- ${ }^{+}$ perivascular fibroblast-like cells that are closely associated with but distinct from vascular-associated NG2 ${ }^{+}$PDGFRB $^{+}$pericytes (Fig. 1n, Supplementary Fig. 2), consistent with the recent description of these cells elsewhere ${ }^{17}$. 
To explore the extent of OPC ablation in greater detail, we generated Pdgfr $\alpha$ CreER $^{\text {T2+/- }}$ : Ail4-tdTomato ${ }^{+/-}$: Sox10-DTA ${ }^{+/-}$mice (hereafter denoted Pdgfr $\alpha^{+}$: $t d T^{+}: D T A^{\dagger}$ ), to enable simultaneous genetic fate-mapping and ablation of OPCs. TAM was administered to 8 -week-old $P d g f r \alpha^{+}: t d T^{+}: D T A^{+}$mice to induce expression of both tdTomato and DTA from the Ai14 tdTomato and Sox10-DTA recombined alleles, respectively (Fig. 2a). Four days after the last dose of TAM, mice received a 6-day intracisternal infusion of $\mathrm{AraC}$ and their brains were collected immediately after infusion to analyze the extent of OPC depletion. Pdgfr $\alpha^{+}: t d T^{+}: D T A^{-}$littermates administered TAM and infused with vehicle alone served as non-ablated controls and also allowed us to determine the specificity and efficiency of tdTomato expression within the intact OPC population.

In non-ablated controls sacrificed at the end of vehicle infusion, $97.0 \pm 0.6 \%$ of PDGFRA ${ }^{+}$OPCs were found to express tdTomato (Fig. 2b,c,e), irrespective of differences in the local density of PDGFRA ${ }^{+}$OPCs along the rostro-caudal axis of the brain (Fig. 2f). We also identified numerous tdTomato ${ }^{+} \mathrm{CC}^{+} \mathrm{OLs}$ generated by these fate-maped OPCs in non-ablated controls (Fig. 2d). By contrast, virtually no tdTomato $^{+}$PDGFRA $^{+}$cells exhibiting typical OPC morphology were detected in the brains of $P d g f r \alpha^{+}: t d T^{+}: D T A^{+}$administered TAM + AraC (Fig. 2b,f, Supplementary Fig. 3). Quantitatively, the mean density of tdTomato ${ }^{+} \mathrm{OPCs}$ in the AraC-infused brains was $99.7 \pm 0.2 \%$ lower than that observed in vehicle-infused brains $(0.16 \pm$ 0.07 cells $/ \mathrm{mm}^{2}$ versus $70.1 \pm 5.8$ cells $\left./ \mathrm{mm}^{2}, p<0.0001\right)$. We also identified significantly fewer non-recombined (tdTomato ${ }^{-}$) OPCs in ablated mice compared to non-ablated controls $\left(0.53 \pm 0.14\right.$ cells $/ \mathrm{mm}^{2}$ versus $2.3 \pm 0.2$ cells $\left./ \mathrm{mm}^{2}, p<0.0001\right)$ (Fig. 2g). By 10 dppr, the mean density of tdTomato ${ }^{+}$OPCs in ablated mice had increased marginally but still remained $97.1 \pm 0.6 \%$ lower than that observed in 
vehicle-infused mice $\left(2.00 \pm 0.93\right.$ cells $/ \mathrm{mm}^{2}$ versus $70.1 \pm 5.8$ cells $\left./ \mathrm{mm}^{2}, p<0.0001\right)$ At this time-point, PDGFRA $^{+}$cells remained depleted in the cerebrum, but began to repopulate caudoventral regions of the brain, particularly the brain stem

(Supplementary Fig. 4). By 20 dppr, PDGFRA ${ }^{+}$cells were evident in both the brain stem and cerebrum (Supplementary Fig. 5). Collectively, our results demonstrate that the use of genetically encoded DTA targeting cells expressing PDGFRA and SOX10 in conjunction with intracisternal infusion of the anti-mitotic agent AraC results in highly efficient OPC ablation throughout the entire brain that persists for at least 10 dppr after which PDGFRA+ cells started to reappear, first in the brain stem then later in the cerebrum.

\section{PDGFRA $^{+}$cells started to repopulate the cerebrum from $12 \mathrm{dppr}$ but did not derive from the OPC lineage}

To examine the kinetics and anatomical origin of PDGFRA ${ }^{+}$cells that repopulated the cerebrum following OPC ablation, additional cohorts of TAM + AraC administered $P d g f r \alpha^{+}: t d T^{+}: D T A^{+}$mice were sacrificed at $0,12,18,20$ and $34 \mathrm{dppr}$. TAM + vehicle administered $P d g f r \alpha^{+}: t d T^{+}: D T A^{-}$mice served as non-ablated controls (Fig 3a). In the corpus callosum of OPC-ablated mice, we confirmed highly efficient ablation of tdTomato ${ }^{+}$PDGFRA $^{+}$OPCs at 0 dppr (Fig. 3b). However, by 20 dppr, we observed high densities of $\mathrm{PDGFRA}^{+}$cells that did not express tdTomato, indicating that the vast majority of these cells do not derive from surviving tdTomato ${ }^{+}$ OPCs (Fig. 3b, Supplementary Fig. 6).

PDGFRA $^{+}$cells repopulating the cerebrum first appeared at $12 \mathrm{dppr}$ in a region of the corpus callosum adjacent to the V-SVZ. Unlike PDGFRA ${ }^{+}$cells found in the corpus callosum of non-ablated controls, those in OPC-depleted mice co- 
expressed nestin, an intermediate filament protein normally expressed by cells in the V-SVZ (Fig. 3c). The PDGFRA ${ }^{+}$cells in OPC-deficient mice also expressed NG2 and GFP, the latter indicating that the Sox10-DTA allele in these cells remained in a non-recombined state (Supplementary Fig. 7). In addition, repopulating PDGFRA ${ }^{+}$ cells were $\mathrm{EdU}^{+}$, indicating that they were born after AraC infusion (data not shown) and many possessed a unipolar or bipolar morphology consistent with migratory activity $^{24}$.

The density of PDGFRA ${ }^{+}$cells in the rostral cerebrum of OPC-depleted mice returned to levels similar to that of non-ablated controls in a spatiotemporally defined manner, normalizing first in the region of the corpus callosum adjacent to the V-SVZ at 12 dppr (Fig. 3d), followed by the midline corpus callosum at $18 \mathrm{dppr}$ (Fig. 3e), and later in the cerebral cortex at $20 \mathrm{dppr}$ (Fig. 3f). Other regions in the rostral cerebrum of OPC-depleted mice took longer for $\mathrm{PDGFRA}^{+}$cell density to return to control levels (Supplementary Fig. 8). Overall, the mean density of PDGFRA ${ }^{+}$cells in the rostral cerebrum returned to levels similar to that of non-ablated controls by 20 dppr whereas caudal regions of the cerebrum remained deficient at the same timepoint (Supplementary Fig. 5b).

Collectively these data demonstrate that ablation of $99.7 \pm 0.2 \%$ of recombined OPCs was followed by a late onset regenerative response resulting in the repopulation of $\mathrm{PDGFRA}^{+}$cells. The finding that the vast majority of repopulating $\mathrm{PDGFRA}^{+}$cells in the cerebrum were tdTomato ${ }^{-}$and $\mathrm{GFP}^{+}$is inconsistent with the notion that PDGFRA ${ }^{+}$cells arise through proliferative expansion of surviving OPCs. Rather, the spatiotemporal pattern of $\mathrm{PDGFRA}^{+}$cell regeneration, emerging first in a region of the corpus callosum adjacent to the V-SVZ, whilst co-expressing the VSVZ marker nestin, raised the possibility that NPCs within the V-SVZ could be the 
primary source of $\mathrm{PDGFRA}^{+}$cells that repopulated this region of the brain following OPC ablation.

\section{Regeneration of V-SVZ-derived NPCs after AraC infusion}

To investigate whether NPCs in the V-SVZ could serve as a reservoir to regenerate PDGFRA ${ }^{+}$cells, we examined the response of NPCs to pharmacogenetic ablation of OPCs. A 6-day infusion of $2 \%$ AraC onto the surface of the brain was previously demonstrated to eliminate rapidly dividing cells in the V-SVZ ${ }^{25}$. The subsequent activation and proliferation of quiescent neural stem cells resulted in complete regeneration of the neurogenic niche including transit-amplifying cells and neuroblasts within 10 days after $\mathrm{AraC}_{\text {withdrawal }}{ }^{25}$. To assess the regeneration of NPCs in TAM + AraC administered Pdgfr $\alpha^{+}: D T A^{+}$mouse brains, mice were given EdU via their drinking water beginning immediately after pump removal and continuing until they were perfused 10 days later (Fig. 1h). Compared to control mice, $\mathrm{DCX}^{+}$neuroblasts were virtually absent from the V-SVZ of AraC-infused mice at 0 dppr. At $10 \mathrm{dppr}$, the number of $\mathrm{DCX}^{+}$cells in the V-SVZ of AraC-treated animals was similar to that of vehicle-treated controls (Fig. 4a,b). In addition, these $\mathrm{DCX}^{+}$cells were positive for EdU (Fig. 4c), indicating that they were born after AraC infusion. We conclude that, consistent with previous reports, the 6-day AraC infusion ablated rapidly dividing NPCs in the V-SVZ but these cells were completely regenerated by $10 \mathrm{dppr}$.

\section{V-SVZ-derived NPCs contributed to regeneration of PDGFRA ${ }^{+}$cells in the dorsal anterior forebrain after OPC ablation}


Our earlier examination of OPC-depleted mice at $12 \mathrm{dppr}$ had revealed that callosal PDGFRA ${ }^{+}$cells adjacent to the V-SVZ co-expressed Nestin, a wellestablished marker of NPCs in the V-SVZ, whereas callosal OPCs in the vehicleinfused controls did not (Fig. 3c). We posited that repopulating PDGFRA ${ }^{+}$cells could derive from progenitors located within the V-SVZ. To explore this question further, we labeled sections from the brains of OPC-deficient mice collected at $20 \mathrm{dppr}$ with antibodies against Nestin, PDGFRA and NG2 and co-stained for EdU and Hoechst. We found $\mathrm{EdU}^{+} \mathrm{PDGFRA}^{+} \mathrm{NG}^{+}$cells in the dorsolateral corner as well as the dorsal and lateral walls of the V-SVZ, suggesting that repopulating PDGFRA ${ }^{+}$cells could originate in the V-SVZ. Interestingly, we found that Nestin was infrequently expressed by EdU ${ }^{+} \mathrm{PDGFRA}^{+} \mathrm{NG}^{+}$cells in the V-SVZ, suggesting that Nestin expression is more prominent at the onset of the regenerative process but is significantly down-regulated by 20 dppr (Supplementary Fig. 9).

To confirm that NPCs generate $\mathrm{PGFRA}^{+}$cells that migrate into the cerebrum following OPC ablation, we developed a novel Dre recombinase-based viral approach for the genetic fate mapping of V-SVZ-derived NPCs (Supplementary Fig. 10). Two weeks prior to pharmacogenetic ablation of OPCs, we injected Pdgfr $\alpha^{+}$:

$t d T^{+}: D T A^{+}$mice with high-titer lentiviruses to transduce NPCs in the V-SVZ (Fig. 5a). This resulted in the expression of a Myc-tagged membrane-targeted mKate2 fluorescent reporter protein by a subset of cells in the V-SVZ (Supplementary Fig. 11). At 20 dppr of AraC, we detected the expression of mKate 2 in a subpopulation of newly-generated PDGFRA ${ }^{+}$cells in the corpus callosum proximal to the V-SVZ, indicating that they derived from the NPC lineage (Fig. 5b,c). 


\section{Effects of OPC ablation on other cell types in the brain}

To confirm whether the induction of DTA-mediated apoptosis was restricted to OPCs, and to exclude the possibility of a bystander effect upon other neural cell types in the brain, we assessed the densities of $\mathrm{CC}^{+}$mature OLs, $\mathrm{ALDH} 1 \mathrm{~L} 1^{+}$ astrocytes, $\mathrm{Iba}^{+}$microglia and $\mathrm{NeuN}^{+}$cortical neurons in coronal brain sections of OPC-ablated and non-ablated controls. Immunohistochemical and histological analyses of brain sections revealed similar OL density and myelin intensity between groups (Fig. 6a-d). Interestingly, further examination of the corpus callosum at 0, 10 and 20 dppr revealed that our pharmacogenetic approach resulted in the loss of both early $\left(\mathrm{PDGFRA}^{+} \mathrm{GPR}^{-} 7^{-}\right.$) and late $\left(\mathrm{PDGFRA}^{+} \mathrm{GPR}^{-} 7^{-}\right.$) OPCs as well as committed oligodendrocyte progenitors (COPs) $\left(\mathrm{PDGFRA}^{-} \mathrm{GPR} 17^{+}\right)$that are in the process of transitioning into mature OL for at least 10 dppr before returning to control levels by 20 dppr (Supplementary Fig. 12). These findings suggest that OPC ablation also caused a transient disruption to the production of new oligodendroglia but did not affect pre-existing mature myelin-forming OLs.

In terms of gliotic responses, we observed moderate and transient astrogliosis and transient activation of microglia 4 days after final TAM administration and at the end of AraC infusion (Fig. 6e-f). These data suggest that extensive OPC loss induced a transient neuroinflammatory response. To assess whether extensive OPC ablation led to neuronal cell death through neuroinflammation as demonstrated in a previous study $^{10}$, NeuN $^{+}$neurons were quantified in the cerebral cortex where PDGFRA ${ }^{+}$cells remained depleted until 20 dppr. Interestingly, the densities of cortical neurons in the cerebral cortex were comparable between groups at all time-points (Fig. 6g) irrespective of cortical layer (data not shown) suggesting that OPC ablation does not compromise the viability of cortical neurons. 


\section{Discussion}

The development of methods to conditionally ablate parenchymal OPCs in the adult mouse CNS provides a powerful experimental paradigm to explore their function. While approaches to ablate adult OPCs have been described ${ }^{6-11}$, the techniques established to date have not allowed for the complete depletion of OPCs throughout the brain. In the absence of complete OPC ablation, surviving OPCs enter the cell cycle and rapidly regenerate their population as a homeostatic response, complicating the interpretation of results. To avert the rapid regeneration by surviving OPCs, we have developed a novel pharmacogenetic approach that allows for the highly efficient ablation of OPCs throughout the brain. This approach consists of genetic elements permitting TAM-dependent induction of DTA expression exclusively in OPCs followed by a pharmacological intervention to prevent repopulation by OPCs that avert genetic targeting. Genetic control of OPC ablation is achieved by crossing the Pdgfra-CreER ${ }^{T 2}$ and Sox10-lox-GFP-STOP-lox-DTA transgenic mouse lines. Double transgenic mice enable highly specific ablation of OPCs at a prescribed time-point due to the dependence of DTA expression upon three levels of regulation: 1) activity of the Pdgfra promoter, 2) activity of the oligodendroglial-specific Sox10 promoter, and 3) temporal control provided by TAMdependent regulation of $\mathrm{CreER}^{\mathrm{T} 2}$ activity. Subsequent intracisternal infusion of the antimitotic drug AraC prevents OPCs that escape genetic targeting from re-entering the cell cycle and repopulating the brain. We found that this pharmacogenetic approach eliminates $99.7 \pm 0.2 \%$ of OPCs throughout the brain without causing any overt adverse effects on the health of animals. 
Despite the highly efficient ablation of OPCs throughout the brain, OPCs were eventually regenerated in the CNS. The pattern and timing of OPC repopulation provides new insight into oligodendrogenic stem and progenitor cell niches in the adult brain. First, we demonstrated that recombined $\left(\right.$ tdTomato $^{+}$) OPCs did not contribute to regeneration in the anterior forebrain. Using lentiviral-mediated delivery of Dre recombinase to target Nestin-expressing cells in the V-SVZ, we revealed that NPCs generate new PDGFRA ${ }^{+} \mathrm{NG}_{2}^{+}$cells that start to repopulate the dorsal anterior forebrain from about day 12 after cessation of AraC infusion. Our findings expand on previous work demonstrating that NPCs originating in the V-SVZ give rise to oligodendrogenic progenitors, both under normal physiological conditions ${ }^{26,27}$ and in response to demyelination of proximal white matter tracts ${ }^{26-29}$. Another study, in which mice undergo global Pdgfra inactivation resulting in OPC depletion, suggested that repopulation of OPCs occurred by expansion of OPCs originating from immature Nestin-expressing cells activated in the meninges and brain parenchyma and from OPCs that escape Pdgfra inactivation ${ }^{11}$. However, spatiotemporal examination of $\mathrm{PDGFRA}^{+} \mathrm{NG}^{+}$cell regeneration after OPC ablation in our model does not provide evidence to support a meningeal origin of newly-generated $\mathrm{PDGFRA}^{+} \mathrm{NG}^{+}$cells (Supplementary Fig. 13).

In the caudoventral brain, we identified extensive repopulation of PDGFRA ${ }^{+}$ cells following ablation, which precedes that observed in the cerebrum. Although, we have not explored the origin of newly-generated PDGFRA ${ }^{+}$cells that emerge in the brain stem, we note that both non-recombined tdTomato ${ }^{-}$PDGFRA $^{+}$cells and recombined tdTomato ${ }^{+} \mathrm{PDGFRA}^{+}$cells contribute to OPC regeneration in the brain stem (Supplementary Fig. 5). These data suggest that some OPCs in the brain stem survive the OPC ablation protocol. This also appears to be the case in the optic nerve 
and spinal cord which also exhibit robust OPC depletion (Supplementary Fig. 14). In the brain stem, it is plausible that repopulating tdTomato ${ }^{-}$PDGFRA $^{+}$cells could arise from immature progenitors residing within alternate stem cell niches located within the brain stem. An oligodendrogenic niche has recently been described in the median eminence of the hypothalamus bordering the third ventricle ${ }^{30}$. Other putative stem cell niches include the ependymal and subependymal zones of the third and fourth ventricles ${ }^{31}$. It is noteworthy that we identified high densities of PDGFRA ${ }^{+}$cells around the aqueduct (Supplementary Fig. 4) and the fourth ventricle (data not shown) at ten days post-pump removal. It thus seems likely that the homeostatic regeneration of $\mathrm{PDGFRA}^{+} \mathrm{NG}^{+}$cells following $\mathrm{OPC}$ depletion is mediated by distinct populations of progenitors in the cerebrum and brain stem.

Collectively, our mouse model of conditional OPC ablation provides a longsought-after methodology to eliminate OPCs in the adult mouse brain. In particular, this study reveals that oligodendrogenic progenitors residing in the V-SVZ play a significant role in the regeneration of PDGFRA ${ }^{+} \mathrm{NG}^{+}$cells in the anterior dorsal forebrain. Future refinement of the model to eliminate the contribution of V-SVZ cells to this homeostatic response could provide an even greater window of opportunity to explore the long-term consequences of OPC ablation upon brain function. 


\section{Figure legends}

\section{Fig. 1: TAM administration followed by intracisternal infusion of AraC resulted} in the ablation of almost all OPCs in the brain.

a, Schematic of the transgenic alleles in $P d g f r \alpha^{+}: D T A^{+}$mice. b, Timeline for the assessment of DTA-mediated OPC ablation after TAM administration. c, Coronal brain sections of a TAM -administered $P d g f r \alpha^{+}: D T A^{-}$mouse immunolabeled with antibodies against GFP and PDGFRA revealed abundant OPCs in the corpus callosum (CC). d, Lack of PDGFRA ${ }^{+}$OPCs in the corpus callosum of a Pdgfr ${ }^{+}: D^{+} A^{+}$mouse sacrificed 4 days after TAM administration. e,f, Regeneration of PDGFRA ${ }^{+}$OPCs at days 8 and 10 after final TAM administration. g, Density of PDGFRA ${ }^{+}$OPCs in the corpus callosum of $P d g f r \alpha^{+}: D T A^{+}$mice at 4, 8 and 10 days after TAM administration. $\mathbf{h}$, Timeline for the assessment of DTA-mediated OPC ablation following TAM + AraC administration. $\mathbf{i}, \mathbf{j}$, OPC repopulation in a vehicle-infused transgenic mouse assessed at the end of infusion (i) or 10 days later (j). k,l, OPC repopulation was not observed in the corpus callosum of an AraC-infused mouse at either 0 or 10 dppr. m,n Residual PDGFRA-expressing cells in an AraC-infused mouse collected at $10 \mathrm{dppr}$ were identified as perivascular fibroblast-like cells based on their close association with PECAM-1-labelled endothelial cells (m) and colocalization of PDGFRA and Laminin-1 (n). CC, corpus callosum; Ctx, cerebral cortex; LV, lateral ventricle; TAM, tamoxifen. Data represent mean \pm SEM.

Statistical analysis: Two-way ANOVA with Dunnett's multiple comparison tests, ${ }^{* *} p<0.01, * * * * p<0.0001$. Scale bars, $70 \mu \mathrm{m}(\mathbf{c - f}, \mathbf{i}-\mathbf{l}), 50 \mu \mathrm{m}(\mathbf{m})$ and $30 \mu \mathrm{m}(\mathbf{n})$. 


\section{Fig 2: Combined fate-mapping and pharmacogenetic ablation revealed highly} efficient and widespread OPC ablation.

a, Schematic of the transgenic alleles in $P d g f r \alpha^{+}: t d T^{+}: D T A^{+}$mice. Following TAM administration to Pdgfr $\alpha^{+}: t d T^{+}: D T A^{+}$mice, DTA and tdTomato expression in recombined OPCs was induced after Cre-mediated excision of the GFP-poly(A) cassette and the STOP cassette, respectively. b, Immunohistochemistry against PDGFRA and tdTomato in the rostral forebrain of transgenic mice assessed at $0 \mathrm{dppr}$. Coronal sections are approximately $+0.25 \mathrm{~mm}$ anterior of Bregma. Compared to nonablated controls, OPC-ablated mice exhibited an almost complete OPC loss. c,d, tdTomato expression in non-ablated mice (TAM + vehicle administered $P d g f r \alpha^{+}$: $t d T^{+}: D T A^{-}$mice) is observed in both PDGFRA ${ }^{+}$OPCs (c, white arrowheads) and in $\mathrm{CC}^{+}$OLs (d, white arrowheads). tdTomato ${ }^{+}$PDGFRA $^{-}$cells (c, yellow arrowheads) are presumptive fate-mapped OPCs that have differentiated into OLs. e, Recombination efficiency of the Ail4 tdTomato allele across the brains of TAM + vehicle administered $P d g f r \alpha^{+}: t d T^{+}: D T A^{-}$mice based on assessment of tdTomato expression among PDGFRA ${ }^{+}$OPCs. f, Cell densities of tdTomato ${ }^{+}$OPCs in coronal $^{-}$ brain sections isolated from non-ablated control versus OPC-ablated mice at various rostrocaudal positions relative to Bregma. g, Densities of tdTomato ${ }^{-}$OPCs in coronal sections of non-ablated and OPC-ablated mice assessed at various rostrocaudal positions at 0 dppr. Data represent mean \pm SEM. Statistical analysis: two-way ANOVA with Bonferroni's post hoc analysis (f,g); ${ }^{*} p<0.05,{ }^{* *} p<0.01, * * * p<0.001$, $* * * * p<0.0001$. Scale bars, $500 \mu \mathrm{m}(\mathbf{b}), 100 \mu \mathrm{m}(\mathbf{c}), 50 \mu \mathrm{m}(\mathbf{d})$. 


\section{Fig. 3: PDGFRA ${ }^{+}$cells that repopulate the corpus callosum following OPC} ablation are not derived from non-ablated fate-mapped OPCs.

a,b, Immunohistochemical detection of $\mathrm{GFP}^{+}, \mathrm{PDGFRA}^{+}$and tdTomato ${ }^{+}$cells in coronal sections of the anterior forebrain of non-ablated versus OPC-ablated mice for 20 dppr. In vehicle-infused controls that do not carry the Sox10-DTA allele, the vast majority of PDGFRA ${ }^{+}$OPCs co-express tdTomato (white arrowheads), reflecting high recombination efficiency of the Ail4 tdTomato allele (a). In addition, the tdTomato $^{+}$PDGFRA $^{-}$cells (yellow arrowheads) are presumptive fate-mapped OPCs that have differentiated into OLs following TAM administration. b, Fate-mapped OPCs expressing PDGFRA and tdTomato were not detected at 0 dppr in TAM + AraC administered Pdgfr $\alpha^{+}: t d T^{+}: D T A^{+}$mice. b, By 20 dppr, PDGFRA $^{+}$cells predominately repopulated the corpus callosum proximal to the SVZ and did not express tdTomato suggesting that they do not derive from the OPC lineage. c, Virtually all PDGFRA ${ }^{+}$cells in the corpus callosum proximal to the V-SVZ expressed the NPC marker Nestin at the initial stage of regeneration at $12 \mathrm{dppr}$ of AraC. High magnification of the boxed region reveals a GFP ${ }^{+}$PDGFRA $^{+}$cell expressing Nestin. d-f, Plots of PDGFRA ${ }^{+}$cell density in the region of the corpus callosum adjacent to the V-SVZ (d), at the midline of the corpus callosum (e) and in the cerebral cortex (f) at the assessed time-points. CC, corpus callosum; LV, lateral ventricle. Data represent mean \pm SEM. Statistical analysis: $* p<0.05, * * * * p<0.0001$, two-way ANOVA with Bonferroni's post hoc analysis. CC, corpus callosum; LV, lateral ventricle. Scale bars, $40 \mu \mathrm{m}(\mathbf{b}, \mathbf{c})$ and $100 \mu \mathrm{m}(\mathbf{d})$. 


\section{Fig. 4: The response of V-SVZ-derived NPCs to AraC treatment.}

a, Expression of the neuroblast marker doublecortin (Dcx) in the V-SVZ of Pdgfr $\alpha^{+}$: $D T A^{+}$mice administered either TAM + vehicle or TAM + AraC and assessed at 0 or 10 dppr. b, Densities of Dcx $^{+}$cells in the V-SVZ of TAM + vehicle or TAM + AraC administered $P d g f r \alpha^{+}: D T A^{+}$mice assessed at 0 or $10 \mathrm{dppr}(\mathrm{n}=4$ mice per group, mean \pm SEM). $(* * * * p<0.0001$, two-way ANOVA with Bonferroni’s post hoc analysis.) c, Coronal sections of the V-SVZ immunolabeled with antibodies against GFP and Dcx, and stained for EdU, which was administered to transgenic mice for 10 dppr. EdU ${ }^{+}$neuroblasts defined by co-expression of Dcx were restricted to the VSVZ. CC, corpus callosum; LV, lateral ventricle. Scale bars, $100 \mu \mathrm{m}$ (a) and $200 \mu \mathrm{m}$ (c).

Fig. 5: V-SVZ-derived NPCs were responsible for the regeneration of new PDGFRA $^{+}$cells in the anterior/dorsal forebrain after OPC ablation.

a, Schematic diagram and timeline of intraventricular injection of lentiviruses to map the fate of V-SVZ-derived NPCs in adult $P d g f r \alpha^{+}: t d T^{+}: D T A^{+}$mice. Schematic diagram was created by BioRender. b,c, In Pdgfr $\alpha^{+}: t d T^{+}: D T A^{+}, \mathrm{TAM}+\mathrm{AraC}$ mice assessed at $20 \mathrm{dppr}$, immunostaining with antibodies against PDGFRA, GFP and mKate 2 revealed some transduced newly-generated PDGFRA ${ }^{+}$cells (white arrowed) expressing membrane-targeted mKate 2 in the corpus callosum proximal to the $\mathrm{V}$ SVZ, suggesting they were derived from the NPC lineage. Scale bars, $60 \mu \mathrm{m}$ (b) and $30 \mu \mathrm{m}(\mathbf{c})$. 


\section{Fig. 6: Cellular consequences of OPC ablation.}

a, Coronal sections of the rostral corpus callosum of TAM-administered Pdgfr $\alpha^{+}$:

$D T A^{+}$mice were immunostained with antibodies against GFP and CC1. b, Densities of $\mathrm{CCl}^{+} \mathrm{GFP}^{+}$cells in the rostral segment of forebrain revealed no effect of OPC ablation upon mature OLs at the assessed time-points. c, Black-Gold II-stained coronal sections of the rostral corpus callosum of vehicle- or AraC-infused mice at days 0, 10 and 20 post pump removal. d, Quantification of the mean Black-Gold II myelin intensity in the entire corpus callosum revealed no effect of OPC ablation on myelination. e, Densities of ALDH1L1 ${ }^{+}$astrocytes in the rostral segment of forebrain revealed no non-specific effect of OPC ablation upon astrocytes although a nonsignificant increase in the number of activated astrocytes was observed at the end of AraC infusion, particularly in the CC. $\mathbf{f}$, Densities of $\mathrm{Iba}^{+}{ }^{+}$cells in the rostral corpus callosum before or after TAM administration, as well as in TAM-administered Pdgfr $\alpha^{+}: D T A^{+}$mice infused either with vehicle or AraC and assessed at days 0,10 and $20 \mathrm{dppr}\left({ }^{*} p<0.05, * * * p<0.001\right.$, unpaired two-tailed Student's $t$ test $) . \mathbf{g}$, Densities of $\mathrm{NeuN}^{+}$cells in the rostral cerebral cortex revealed no effect of OPC ablation on cortical neurons. Sample size, $n=4-7$ mice per group, mean \pm SEM (b,d,e-g). CC, corpus callosum; LV, lateral ventricle. Scale bars, $200 \mu \mathrm{m}$ (a) and $150 \mu \mathrm{m}(\mathbf{c})$. 


\section{Supplementary information.}

\section{Supplementary Fig. 1: DTA-mediated ablation of OPCs induced rapid cell} regeneration.

a,b, All residual $\mathrm{GFP}^{+}$cells in $P d g f r \alpha^{+}: D T A^{+}$mice examined 4 days after TAM administration expressed Sox10 and CC1 (yellow arrowheads). A subpopulation of Sox $10^{+}$oligodendroglia did not express GFP (a, white arrowheads) suggesting that the Sox10-lox-GFP-STOP-lox-DTA allele is not transcriptionally active in all Sox $10^{+}$ oligodendroglia. c, EdU labeling of PDGFRA ${ }^{+}$cells in $P d g f r \alpha^{+}: D T A^{+}$mice at 8 days post TAM administration. d, Densities of PDGFRA ${ }^{+}$OPCs and their subpopulations expressing GFP and/or EdU in the corpus callosum of $P d g f r \alpha^{+}: D T A^{+}$mice at 4,8 and 10 days after the final tamoxifen administration ( $n=4$ mice per group). Data represent mean \pm SEM. Statistics: Two-way ANOVA with Tukey’s multiple comparison tests, ${ }^{*} p<0.05, * * * * p<0.0001$ Scale bars, $50 \mu \mathrm{m}$ (a) and $40 \mu \mathrm{m}(\mathbf{b}, \mathbf{c})$.

\section{Supplementary Fig. 2: PDGFRA-expressing cells associated with the brain} vasculature are perivascular fibroblast-like cells.

a-c, Immunohistochemistry against PDGFRA, PECAM-1, Laminin-1, NG2 and PDGFRB in coronal sections reveals vascular-associated PDGFRA ${ }^{+}$cells are perivascular fibroblasts-like cells surrounding PECAM- $1^{+}$endothelial cells. Colocalization analysis of PDGFRA with Laminin-1, a key vascular basement membrane component (a) and pericyte markers NG2 and PDGFRB (b,c) in a TAM + vehicle administered $P d g f r \alpha^{+}: D T A^{-}$mouse $(\mathbf{a}, \mathbf{b})$ and a TAM + AraC administered Pdgfr $\alpha^{+}: D T A^{+}$mouse (c). Frequency scatterplots of fluorescence intensity in the red and green channels and photomicrographs showing colocalized red and green pixels (bottom right panels, in white) revealed a high degree of colocalization between 
PDGFRA and Laminin-1 (a',a”) whereas the degree of colocalization between PDGFRA and NG2 (b',b”) or PDGFRA and PDGFRB (c',c'”) was low. d, Plot of Pearson's r coefficient of colocalization between PDGFRA and other antigens. ****p<0.0001, one-way ANOVA with Bonferroni's post hoc analysis. Scale bars, 20 $\mu \mathrm{m}(\mathbf{a})$ and $30 \mu \mathrm{m}(\mathbf{b}, \mathbf{c})$.

\section{Supplementary Fig. 3: Intracisternal infusion of AraC following TAM-induced DTA expression in OPCs results in highly efficient OPC ablation throughout the brain.}

a, Immunohistochemical detection of PDGFRA in serial coronal brain sections collected from non-ablated controls (upper row) and OPC-ablated mice (lower row) at the end of AraC or vehicle infusion ( $0 \mathrm{dppr})$. The non-ablated control group comprised Pdgfr $\alpha^{+}: t d T^{+}: D T A^{-}$mice administered TAM + vehicle. The OPC-ablated group consisted of $P d g f r \alpha^{+}: t d T^{+}: D T A^{+}$mice administered TAM + AraC. The approximate position along the rostro-caudal axis for each coronal section is indicated below the photomicrographs. $\mathbf{b}$, High magnification of the boxed region from (a) reveals that remnant PDGFRA $^{+}$cells in OPC-deficient mice do not exhibit typical OPC morphology and are localized around PECAM-1 ${ }^{+}$endothelial cells within the brain vasculature. c, Densities of tdTomato ${ }^{+}$PDGFRA $^{+}$perivascular cells in coronal sections of non-ablated and OPC-ablated mice assessed at various rostrocaudal positions at 0 dppr. Data represent mean \pm SEM. Statistical analysis: two-way ANOVA with Bonferroni's post hoc analysis; ${ }^{*} p<0.05,{ }^{* *} p<0.01$. Scale bars, $900 \mu \mathrm{m}$ (a) and $100 \mu \mathrm{m}$ (b). 


\section{Supplementary Fig. 4: PDGFRA-expressing cells remain depleted in the cerebrum for at least 10 days post infusion of AraC.}

Immunohistochemistry against PDGFRA on coronal brain sections of a TAM + AraC-administered $P d g f r \alpha^{+}: D T A^{+}$mouse at 10 dppr reveals that ramified PDGFRA ${ }^{+}$ cells remained depleted in the cerebrum $(\mathrm{CH})$ but had started to repopulate more caudoventral regions of the brain such as brain stem (BS). The approximate rostrocaudal position relative to Bregma is indicated below each photomicrograph. Scale bar, $1 \mathrm{~mm}$.

\section{Supplementary Fig. 5: Regeneration of PDGFRA-expressing cells in the brain} within 20 days post infusion of AraC.

a, Immunohistochemistry against PDGFRA on coronal brain sections of a TAM + AraC-administered $P d g f r \alpha^{+}: D T A^{+}$mouse examined at 20 dppr reveals significant repopulation of both the cerebrum and brain stem with PDGFRA+ cells. The approximate rostro-caudal position relative to Bregma is indicated below each photomicrograph. b, $P d g f r \alpha^{+}: D T A^{+}$mice administered TAM + AraC and assessed at 10 dppr revealed that unlike dorsal regions of the cerebrum where OPCs remained depleted, PDGFRA-expressing cells in the caudoventral brain such as brain stem were regenerated. By 20 dppr, only dorsal-caudal regions were not fully repopulated by newly-generated cells $(* * * * p<0.0001$ compared to non-ablated TAM + vehicle $(0$ dppr) group, two-way ANOVA with Bonferroni's post hoc analysis). c, Densities of PDGFRA $^{+}$only and PDGFRA ${ }^{+}$tdTomato $^{+}$cells in the brain at 0 and $20 \mathrm{dppr}$. BS, brain stem; $\mathrm{CH}$, cerebrum. Scale bar, $1 \mathrm{~mm}$. 


\section{Supplementary Fig. 6: PDGFRA ${ }^{+}$cells were predominately repopulated in the} corpus callosum proximal to the V-SVZ in the anterior forebrain.

a, Coronal section of the rostral forebrain of a $P d g f r \alpha^{+}: t d T^{+}: D T A^{-}$mouse administered TAM + vehicle at $20 \mathrm{dppr}$, tdTomato was expressed in recombined PDGFRA $^{+}$OPCs and in oligodendroglia that have differentiated from fate-mapped OPCs, as well as in some PDGFRA ${ }^{+}$perivascular fibroblast-like cells. b, By contrast, Pdgfr $\alpha^{+}: t d T^{+}: D T A^{+}, \mathrm{TAM}+$ AraC mice at 20 dppr exhibited repopulation of PDGFRA $^{+}$cells, with the highest cell density in the corpus callosum proximal to the V-SVZ and lower densities in the septum, caudate putamen and cerebral cortex.

Interestingly, tdTomato expression was not observed in these regenerated cells and it was only expressed in non-ablated perivascular fibroblast-like cells. c, Densities of PDGFRA $^{+}$cell subpopulations in the corpus callosum and cerebral cortex of Pdgfr $\alpha^{+}$: $t d T^{+}: D T A^{-}$and $P d g f r \alpha^{+}: t d T^{+}: D T A^{+}$mice administered TAM + vehicle or TAM + AraC and assessed at either 0 or $20 \mathrm{dppr}(\mathrm{n}=4$ mice per group, mean $\pm \mathrm{SEM}$ ).

$(* * * * p<0.0001$, two-way ANOVA with Bonferroni's post hoc analysis.). CC, corpus callosum; $\mathrm{CPu}$, caudate putamen; $\mathrm{Ctx}$, cerebral cortex; $\mathrm{LV}$, lateral ventricle. Scale bar, $700 \mu \mathrm{m}(\mathbf{a}, \mathbf{b})$.

\section{Supplementary Fig. 7: Regenerated PDGFRA ${ }^{+} \mathrm{NG2}^{+}$cells resembled OPCs in their pattern of protein expression and morphology in the corpus callosum proximal to the V-SVZ.}

Coronal sections of the rostral corpus callosum of an OPC-deficient mouse assessed at $20 \mathrm{dppr}$ and labelled with antibodies against GFP, PDGFRA and NG2. PDGFRA ${ }^{+}$ $\mathrm{NG}^{+}$cells expressing the Sox 10-GFP transgene (white arrows) that exhibited typical OPC-like morphology were identified proximal to the V-SVZ. CC, corpus callosum; 
Ctx, cerebral cortex; LV, lateral ventricle. Scale bars, $100 \mu \mathrm{m}$ and $40 \mu \mathrm{m}$ (boxed).

\section{Supplementary Fig. 8: Spatiotemporal profile of repopulation of newly- generated PDGFRA ${ }^{+}$cells in the anterior forebrain of OPC-depleted mice.}

a, Densities of PDGFRA ${ }^{+}$cells in the septum, nucleus accumbens, caudate putamen and olfactory tubercles revealed re-emergence of PDGFRA $^{+}$cells in a spatiotemporal manner $(\mathrm{n}=4$ mice per group, mean $\pm \mathrm{SEM}),\left({ }^{*} \mathrm{p}<0.05, * * \mathrm{p}<0.01, * * * p<0.001\right.$, $* * * * p<0.0001$, two-way ANOVA with Bonferroni's post hoc analysis). b, Schematic diagram depicting the various brain regions within which $\mathrm{PDGFRA}^{+}$cell densities were quantitated.

\section{Supplementary Fig. 9: Newly-generated PDGFRA-expressing cells were} observed in different spatial domains of the V-SVZ at 20 dppr.

a, Schematic diagram of the fluorophore inactivation (quenching) procedure conducted prior to immunohistochemistry. Sections with intrinsic GFP and tdTomato expression (1) were oxidized using hydrogen peroxide (2) prior to immunohistochemistry. Schematic diagram was created using BioRender. b, Brain sections with intrinsic GFP or tdTomato expression before (left) and after (right) fluorophore inactivation to confirm fluorophore quenching. c-e, Immunostaining on fluorophore-quenched tissues revealed the existence of $\mathrm{EdU}^{+} \mathrm{PDGFRA}^{+} \mathrm{NG}^{+}$cells (white arrowheads) in the dorsolateral (DL-SVZ) (c and $\mathbf{c}^{\prime}$ ), dorsal (D-SVZ) (d) and lateral (L-SVZ) (e) walls of the lateral ventricle of $P d g f r \alpha^{+}: t d T^{+}: D T A^{+}$mice administered TAM + AraC and assessed at 20 dppr. The EdU ${ }^{+}$PDGFRA $^{+} \mathrm{NG}^{+}$cells in the DL-SVZ and D-SVZ did not express Nestin suggesting its downregulation at this time-point (20 dppr), in contrast to the $\mathrm{EdU}^{+} \mathrm{PDGFRA}^{+} \mathrm{NG}^{+}$cell in the $\mathrm{L}-\mathrm{SVZ}$ 
which also expressed Nestin. Scale bars: $90 \mu \mathrm{m}(\mathbf{b}), 25 \mu \mathrm{m}(\mathbf{c}), 35 \mu \mathrm{m}(\mathbf{d})$ and $20 \mu \mathrm{m}$ (e).

Supplementary Fig. 10: Lentiviral approach for the fate-mapping of V-SVZderived NPCs.

a, Schematic map for the construction of the FUW-EF1 $\alpha$-FREX-Myc/mKate2-f-mem plasmid. b, Schematic map for the construction of the FUW-Nestin-NLS-HA-Dre plasmid. c, Upon Dre-mediated recombination, the coding sequence of Myc/mKate2f-mem is flipped into the correct sense orientation resulting in the expression of Myctagged, membrane-targeted mKate 2 under the control of the human EF1 $\alpha$ promoter. d, In vitro validation of plasmids showing HEK293T cells were transfected with commercial pmKate2-f-mem (Evrogen) as a positive control or pNestin-NLS-HA-Dre and $p E F 1 \alpha$-FREX-Myc/mKate2-f-mem. Immunostaining shows mKate2 expression in the transfected HEK293T cells. Nuclei were stained with Hoechst. e, In vivo validation of lentiviral vectors showing the co-expression of mKate2 and Myc-tag or HA-tag in the V-SVZ wall of the lateral ventricle at 2 weeks after intraventricular injection of lentiviruses into wild type $C 57 B L / 6 J$ mice. f,g, mKate2 expression was also observed in transduced multi-ciliated ependymal cells immunoreactive for $\alpha$ SMA (f, yellow arrowed) or FoxJ1 (g) in addition to NPCs (f, white arrowed) in the wild-type V-SVZ. LV, lateral ventricle. Scale bars, $180 \mu \mathrm{m}(\mathbf{d}), 100 \sim 160 \mu \mathrm{m}(\mathbf{e}), 24$ $\mu \mathrm{m}(\mathbf{f})$ and $12 \mu \mathrm{m}(\mathbf{g})$.

Supplementary Fig. 11: Lentiviral-transduced cells expressing Myc-tag in the VSVZ of transgenic mice at 20 days post AraC infusion.

a,b, Membrane-targeted Myc-tag expression was observed in the V-SVZ, including

D-SVZ (a) and DL-SVZ (b). Scale bars, $40 \mu \mathrm{m}$ (a) and $85 \mu \mathrm{m}$ (b). 


\section{Supplementary Fig. 12: The effect of OPC ablation upon cells at intermediate stages of OL differentiation.}

a, Coronal sections of the rostral corpus callosum of a non-ablated control (Pdgfr $\alpha^{+}$: $D T A^{-}, \mathrm{TAM}+$ vehicle $)$ and OPC-ablated mouse $\left(P d g f r \alpha^{+}: D T A^{+}, \mathrm{TAM}+\mathrm{AraC}\right)$ collected at 0 and 20 dppr and immunostained with antibodies against PDGFRA and GPR17. b, Densities of cells expressing PDGFRA and/or GPR17 in the rostral corpus callosum at the assessed time-points. Early OPCs $\left(\mathrm{PDGFRA}^{+} \mathrm{GPR}^{-} 7^{-}\right.$and $\mathrm{PDGFRA}^{+}$ GPR $17^{+}$) and intermediate pre-OLs (PDGFRA ${ }^{-}$GPR $17^{+}$) were completely ablated at both 0 and 10 dppr ( $n=4$ mice per group, mean \pm SEM). CC, corpus callosum; LV, lateral ventricle. Scale bar, $75 \mu \mathrm{m}(\mathbf{a})$

\section{Supplementary Fig. 13: No evidence of newly-generated PDGFRA ${ }^{+}$cells from the vicinity of the meningeal membrane.}

a, Immunohistochemistry against PDGFRA, Laminin-1 and GFP on a coronal section of the cerebral cortex of a $P d g f r \alpha^{+}: t d T^{+}: D T A^{+}$mouse administered TAM + AraC and collected at 20 dppr. a', High magnification of the boxed region shown in (a) reveals no evidence of PDGFRA ${ }^{+}$cell regeneration near the meninges. a', Whereas in the deeper cortical region, a few newly-generated PDGFRA ${ }^{+}$cells were observed. b, Representative coronal section from the cerebral cortex of a $P d g f r \alpha^{+}: t d T^{+}: D T A^{+}$ mouse administered TAM + AraC and collected at 20 dppr was immunostained with antibodies against PDGFRA, NG2 and Nestin, as well as Hoechst and EdU after fluorophore inactivation. The image demonstrates the absence of newly-generated PDGFRA $^{+} \mathrm{NG}^{+} \mathrm{EdU}^{-}$cells near the meninges. Scale bars, $100 \mu \mathrm{m}(\mathbf{a})$ and $40 \mu \mathrm{m}$ (b). 


\section{Supplementary Fig. 14: Ablation and regeneration of PDGFRA $^{+}$cells in the coronal spinal cord and optic nerve.}

a, In TAM + vehicle administered Pdgfr $\alpha^{+}: t d T^{+}: D T A^{-}$mice, tdTomato was expressed in the majority of PDGFRA ${ }^{+}$OPCs and in oligodendroglia that have differentiated from fate-mapped OPCs. b, Fate-mapped OPCs were ablated in the coronal spinal cord and optic nerve of TAM + AraC administered Pdgfr $\alpha^{+}$:

$t d T^{+}: D T A^{+}$mice and assessed at 0 dppr. By 20 dppr, PDGFRA ${ }^{+}$cells had regenerated with a mixture of tdTomato-positive and tdTomato-negative cells. c,d, Densities of PDGFRA $^{+}$cells in the coronal spinal cord (c) and optic nerve (d) of Pdgfr $\alpha^{+}: t d T^{+}$: $D T A^{-}$and $P d g f r \alpha^{+}: t d T^{+}: D T A^{+}$mice administered TAM + vehicle or TAM + AraC and assessed at either 0 or $20 \mathrm{dppr}(\mathrm{n}=3-4$ mice per group, mean $\pm \mathrm{SEM}),\left({ }^{*} p<0.05\right.$, $* * p<0.01, * * * p<0.001, * * * p<0.0001$, two-way ANOVA with Tukey's post hoc analysis). CSp, coronal spinal cord; OpN, optic nerve. Scale bars, $250 \mu \mathrm{m}$ (Csp), 100 $\mu \mathrm{m}(\mathrm{OpN})$. 


\section{Methods}

Animals. Animal experiments were conducted in accordance with the National Health and Medical Research Council guidelines for the care and use of animals. All animal studies were approved by the animal ethics committee of the Florey Institute of Neuroscience and Mental Health (Parkville, VIC, Australia) and the animal ethics committee of Monash University (Clayton, VIC, Australia). Pdgfr $\alpha-C r e E R^{T 2}$ BAC transgenic mice expressing $\mathrm{CreER}^{\mathrm{T} 2}$ under the regulation of the Pdgfra gene promoter, and Sox10-DTA transgenic mice expressing a P1-derived artificial chromosome DNA construct containing the gene cassette Sox10-lox-GFP-poly(A)lox-DTA driven by the Sox10 promoter, were kindly provided by Prof. William Richardson (University College London, UK) ${ }^{14,16}$. These two mouse lines were crossed to generate $P d g$ fr $\alpha-C r e E R^{T 2+/+}:$ Sox $10-D T A^{+/-}$and $P d g f r \alpha-C r e E R^{T 2+/+}$ : Sox 10-DTA ${ }^{-1-}$ breeders that were used to produce experimental cohorts. To generate Pdgfr $\alpha-\mathrm{CreER}^{T 2+/-}:$ Sox10-DTA ${ }^{+/-}:$Ail4 tdTomato $^{+/-}$mice, we crossed Pdgfr $\alpha$ CreER $^{T 2+/+}$ : Sox10-DTA ${ }^{+/-}$mice with homozygous Ail4 tdTomato $^{+/+}$mice, which were purchased from the Jackson Laboratory.

Tamoxifen induction. Cre-mediated recombination was induced by oral gavage of tamoxifen (Sigma) delivered at a dose of $0.3 \mathrm{~g} / \mathrm{kg} / \mathrm{d}$ for 4 consecutive days. Tamoxifen was prepared at $40 \mathrm{mg} / \mathrm{mL}$ in corn oil (Sigma).

EdU administration. To label cells that proliferated during the first 10 days following AraC withdrawal, 5-ethynyl-2'-deoxyuridine (EdU; Life Technologies) was 
administered to mice in their drinking water at $0.1 \mathrm{mg} / \mathrm{mL}$. EdU-supplemented drinking water was placed in light-proof water bottles and replaced every 3 days.

Preparation of AraC for intracisternal infusions. Cytosine- $\beta$-D-arabinofuranoside (AraC, Sigma) was prepared at a final concentration of $2 \%(\mathrm{w} / \mathrm{v})$ in artificial CSF (aCSF, Tocris Bioscience). One hundred microliters of either $2 \%$ AraC or vehicle (aCSF) was injected into osmotic minipumps (Alzet Model 1007D, flow rate 0.5 $\mu \mathrm{L} /$ hour, Brain Infusion Kit III) using a $1 \mathrm{~mL}$ syringe attached to a blunt fill needle. The flow moderator was attached to a bespoke tubing assembly made by connecting PE-10 polyethylene tubing to the vinyl catheter tube provided with the Brain Infusion Kit III (Alzet). The flow moderator with attached tubing was slowly inserted into the filled osmotic minipump to create a complete pump assembly. The pumps were then transferred into $50 \mathrm{~mL}$ conical tubes containing sterile saline and placed in a $37^{\circ} \mathrm{C}$ water bath overnight to prime the pumps prior to surgical implantation.

Surgical implantation of osmotic minipumps. AraC or vehicle (aCSF) was infused into the CSF at the level of the cisterna magna via an osmotic minipump for a period of 6 days. Prior to anesthesia, mice received a subcutaneous injection of meloxicam (2 $\mathrm{mg} / \mathrm{kg}, 0.25 \mathrm{~mL} / 10 \mathrm{~g}$ body weight) in warm saline. Mice were then anaesthetized by isoflurane inhalation (4\% induction, $2 \%$ maintenance). The head of the anesthetized mouse was fixed in a stereotaxic frame using a nose cone and ear bars. The position of the abdomen was lowered so that the neck was flexed at an angle of 30 45 degrees relative to horizontal and the body was placed on a thermostatically controlled heat pad to maintain body temperature. Eyes were moistened with water-based lubricant and the fur was cleared over the head and shoulders with an electric shaver. A sterile 
cotton tip soaked in $80 \%$ ethanol was used to swab and clean the surface of incision site, followed by $10 \%$ (w/v) povidone-iodine solution (Betadine). A midline skin incision was made using a sharp scalpel from a position just rostral of the external occipital protuberance to $\sim 1 \mathrm{~cm}$ cranial to the shoulders. The atlanto-occipital membrane was visualized after blunt dissection of the muscle layers to expose the position of the cisterna magna. Using a straight hemostat, a pocket was created by spreading the subcutaneous connective tissues apart and the osmotic minipump was inserted into the pocket. The cisterna magna was pierced superficially with a $30 \mathrm{G}$ needle and the PE-10 tubing connected to the osmotic minipump was introduced into the hole before applying a small amount of superglue to fix the tubing in place. The position of the tubing was further anchored and fixed to the musculature using sutures. Bupivacaine (100 $\mu \mathrm{L}$ of $0.25 \%$ solution) was flushed over the musculature to provide rapid onset analgesia. The skin was then sutured and $10 \%(\mathrm{w} / \mathrm{v})$ povidoneiodine solution was applied to the sutured skin. The animal was placed in a warm recovery box for monitoring until it regained consciousness and normal mobility. Animals were monitored daily throughout the experiment and were administered meloxicam ( $2 \mathrm{mg} / \mathrm{kg}, 0.25 \mathrm{~mL} / 10 \mathrm{~g}$ body weight) in warm saline once daily for the first 2 days post-surgery. All mice were provided with powdered chow mixed with fresh water daily in a small dish that was easily accessed within the animal cage.

Surgical removal of osmotic minipumps. Infusion of $\mathrm{AraC}$ or vehicle (aCSF) was ceased after 6 days by removing the osmotic minipumps. Animals were anaesthetized as described above and the former skin incision site was reopened to gain access to the tubing connected to the osmotic minipump. The tubing was cut $2 \mathrm{~mm}$ from the glued/sutured musculature and the minipump was removed from the pocket. The 
tubing fixed to the musculature was left in place and the free end of the tubing was sealed with superglue. The incision was closed with sutures and the animal was placed in a warm recovery box for monitoring until it regained consciousness and normal mobility. Animals were monitored daily throughout the experiment. AraCadministered mice experienced a mild reduction in body weight during AraC infusion. If mice showed signs of greater than $10 \%$ weight loss, they were given powdered chow mixed with fresh water daily in a small dish that was easily accessed within the animal cage. If mice maintained greater than $15 \%$ weight loss for more than 72 hours, they were humanely euthanized. Following removal of the osmotic minipumps delivering AraC, mice returned to normal weight.

Generation of lentiviral vectors. The lentiviral vectors $L V$-FUW-Nestin-NLS-HADre and $L V$-FUW-EF1 $\alpha$-FREX-Myc/mKate2-f-mem were used for fate-mapping of VSVZ-derived NPCs. These vectors were constructed using standard molecular cloning techniques, including PCR using Phusion DNA polymerase (New England Biolabs), restriction enzyme digestion and Gibson assembly. To create these lentiviral vectors, the rat Nestin promoter sequence was amplified by PCR from plasmid DNA (Addgene plasmid \#32401). The DNA encoding NLS-HA-Dre was amplified by PCR from plasmid DNA (Addgene plasmid \#51272). The coding sequence for the rat Nestin second intron enhancer was amplified from rat genomic DNA. EF1 $\alpha$ promoter sequence was amplified by PCR from plasmid DNA (Addgene plasmid \#38770). The FREX-Myc/mKate2-f-mem DNA sequence was generated by DNA synthesis (Integrated DNA Technologies). The PCR products were cloned into the FUGW lentiviral vector backbone (Addgene plasmid \#14883) in place of the GFP coding sequence by Gibson Assembly (New England Biolabs). Plasmid DNA was then 
extracted and purified using Plasmid Mini or Midi Kits (Qiagen). Sequences of the vector constructs were verified by DNA sequencing (Micromon, Monash University).

In vitro validation of lentiviral vectors. Plasmid DNA was transfected into HEK293T cells cultured at $37^{\circ} \mathrm{C}$ and $5 \% \mathrm{CO}_{2}$ and analyzed $48 \mathrm{~h}$ post-transfection for fluorescence. The pmKate2-f-mem plasmid (Evrogen) served as a positive control for mKate2 fluorescence. HEK293T cells were plated in a 24 well plate and cultured in Dulbecco's Modified Eagle's Medium (DMEM, Gibco), supplemented with 10\% fetal bovine serum (Invitrogen). At $80 \%$ confluency, cells were transfected with the plasmids using Lipofectamine 2000 Transfection Reagent (Invitrogen) according to the manufacturer's instructions. The growth medium was replaced with fresh medium containing $100 \mathrm{U} / \mathrm{mL}$ penicillin and $100 \mathrm{mg} / \mathrm{mL}$ streptomycin (Gibco) $4 \mathrm{~h}$ post transfection. At $48 \mathrm{~h}$ after transfection, cells were post-fixed with 4\% PFA/DPBS, processed for immunocytochemistry and imaged for mKate 2 expression using a Zeiss LSM780 confocal microscope.

Lentivirus production. Lentiviruses were produced and packaged in HEK293T cells by the Vector and Genome Engineering Facility, Children's Medical Research Institute. To determine viral titer, HEK293T cells were transduced with lentivirus. Genomic DNA was extracted from transduced cells and viral DNA copy number/cell was determined by Multiplex Taqman qPCR.

Intraventricular injection of lentiviral vectors. Mice were anaesthetized by isoflurane inhalation and positioned in a motorized stereotaxic frame as described above. A small incision was made in the scalp and the injection site of the lateral 
ventricle was marked using the following stereotaxic coordinates, relative to Bregma: anterior-posterior $-0.22 \mathrm{~mm}$, medial-lateral $-1.00 \mathrm{~mm}$, and dorsal-ventral $-2.5 \mathrm{~mm}$. A small hole was drilled into the skull to expose the brain surface. Five microliters of mixed lentiviral vectors was gently drawn up into a blunt-tipped 35G needle attached to a $10 \mu \mathrm{L}$ NanoFil syringe (World Precision Instruments, WPI). The syringe was then placed into a microinjection pump (UltraMicroPump 4, WPI) attached to the stereotaxic frame and lowered slowly into the injection site of the lateral ventricle according to the dorsal-ventral coordinate. The microinjection pump controlled the infusion of $5 \mu \mathrm{L}$ total volume of lentiviruses at a flow rate of $0.5 \mu \mathrm{L} / \mathrm{min}$, after which the needle was left in place for 5 min to ensure complete diffusion of the viruses and avoid backflow.

Tissue processing and immunohistochemistry. Mice were deeply anesthetized with $100 \mathrm{mg} / \mathrm{kg}$ sodium pentobarbitone and then transcardially perfused with PBS, followed by $4 \%$ PFA/PBS. Brains, optic nerves and spinal cords were removed and post-fixed in $4 \%$ PFA/PBS for $2 \mathrm{~h}$ on ice, transferred to PBS overnight, cryopreserved in $20 \%$ sucrose/PBS overnight, followed by embedding in Tissue-Tek OCT compound (Sakura FineTek). The tissues were stored at $-80^{\circ} \mathrm{C}$ until sectioned. Ten micron-thick coronal sections of the brain and spinal cord were cut on a Leica cryostat, collected onto Superfrost Plus slides (Menzel Glaser), and air dried for $1 \mathrm{~h}$ before storing at $-80^{\circ} \mathrm{C}$ until stained. Cryosections were air dried, then blocked with PBS containing $0.3 \%$ Triton X-100, 10\% normal donkey serum, and 10\% BlokHen (Aves Labs) for $1 \mathrm{~h}$ at room temperature (RT). The sections were then incubated with primary antibodies at RT overnight, followed by $1 \mathrm{~h}$ incubation at RT with secondary antibodies. 
For multiplex immunohistochemistry, some primary antibodies were incubated simultaneously. The following primary antibodies were used: mouse antiAPC/CC1 (1:100; Calbiochem), goat anti-PDGFRA (1:150; R\&D Systems), rat antiPDGFRA (1:150; BD Pharmingen), rabbit anti-ALDH1L1 (1:1000; Abcam), rabbit anti-NG2 (1:200; Millipore), rabbit anti-Laminin-1 (1:400, Sigma), goat anti-SOX10 (1:100, R\&D Systems), goat anti-DCX (1:100; Santa Cruz Biotechnology), goat antiPDGFRB (1:200, R\&D Systems), rabbit anti-Ibal (1:200; Wako), mouse anti-NeuN (1:100; Millipore), mouse anti-Nestin (1:100; Millipore), rabbit anti-GPR17 (1:800, Cayman), rabbit anti-RFP for mKate2 labelling (1:500, Kerafast), mouse anti-Myc$\operatorname{tag}(1: 500$, Sigma), mouse anti-HA-tag (1:500, Sigma), mouse anti- $\alpha-$ SMA (1:500, Abcam), mouse anti-FoxJ1 (1:200, eBioscience) and chicken anti-GFP (1:2000; Aves Labs). To label $\mathrm{GFP}^{+}$tdTomato $^{+}$brain sections using primary antibodies against Nestin, PDGFRA, and NG2, as well as EdU and Hoechst, inactivation of GFP and tdTomato was performed by firstly treating the brain sections with $3 \% \mathrm{H}_{2} \mathrm{O}_{2}$ and 20 $\mathrm{mM} \mathrm{HCl}$ in PBS for $1 \mathrm{~h}$ at $\mathrm{RT}$ with light illumination ${ }^{28}$. The slides were then washed 3 times with PBS, incubated with blocking buffer and processed for the immunostaining as described above.

Secondary antibodies raised in donkey and conjugated to Alexa Fluor 488, FITC, TRITC, Alexa Fluor 594 or Alexa Fluor 647 were purchased from Jackson ImmunoResearch or Invitrogen and used at 1:200 dilution. Sections incubated with biotinylated rat anti-PECAM1/CD31 antibody (1:200; BD Biosciences) were rinsed and further incubated with streptavidin-Brilliant Violet 480 (1:200; BD Biosciences) for $30 \mathrm{~min}$. Some slides stained without the fluorophore Brilliant Violet 480 were also counterstained with Hoechst $33342(1 \mu \mathrm{g} / \mathrm{mL}$; Invitrogen).

For myelin analysis, slides were stained with Black-Gold II (Biosensis) 
according to the manufacturer's instructions. To detect EdU incorporation in proliferating cells, sections were first processed for immunohistochemistry as above, followed by EdU detection using the Click-iT EdU Alexa Fluor 647 Imaging Kit (Life Technologies) as per the manufacturer's instructions. Sections were coverslipped with Mowiol mounting medium and subjected to fluorescence and confocal microscopic analysis.

\section{Fluorescence imaging and image analysis.}

Stained $10 \mu \mathrm{m}$ thick coronal sections were imaged by laser scanning confocal microscopy (Zeiss LSM510-META or Zeiss LSM780), which was used to detect up to four fluorophores by laser excitation at 405, 488, 561 and $633 \mathrm{~nm}$ wavelengths. For five-color imaging such as brain sections stained with Brilliant Violet 480 or Hoechst, as well as Alexa Fluor 488, TRITC, Alexa Fluor 594 and Alexa Fluor 647, linear unmixing was performed during acquisition (online fingerprinting). Tile scanning was performed at a magnification of 10x or 20x for the cellular analysis of the entire brain sections of transgenic mouse lines. Confocal images were then imported into ImageJ for quantification of cellular density in the regions of interest.

For global analysis of cellular distribution in the entire mouse brain across Bregma levels, 2-3 random sections were scanned from the stained slides on an Olympus VS120 Virtual Slide Microscope with a 20x objective at Monash Histology Platform, Monash University. The resulting images have a pixel resolution of $0.65 \mu \mathrm{m} / \mathrm{pixel}$. Double- or triple-positive cells from these images were automatically counted using ImageJ scripts created by Monash Micro Imaging, Monash University. Results obtained from automatic cell quantification were also validated by manual counts. For 
the analysis of Laminin-1/PDGFRA, NG2/PDGFRA or PDGFRB/PDGFRA colocalization, confocal images were acquired using a $63 \mathrm{X}$ objective to generate $\mathrm{z}$ stacks. Complete image stack deconvolution was performed with the "Iterative Deconvolve 3D” plugin in ImageJ. Colocalization analysis was performed by using the "Colocalization threshold" plugin in ImageJ to automatically determine a detection threshold for each channel to avoid subjective bias. The extent of withinpixel fluorescent signal colocalization as indicated by Pearson's correlation coefficient was calculated in each optical slice and then flattened into a maximal zprojection to reveal colocalized pixels across the entire image thickness. All analyses were performed in a blinded fashion.

Bright-field imaging and image analysis. For the sections stained with Black-Gold II, 2-3 representative images were taken using a 10x objective on a Zeiss Axioplan upright fluorescent microscope and captured with an Axioplan HRc camera (Carl Zeiss) using the Axiovision 7.2 imaging software. All images were taken with the same exposure time. For quantification of myelin intensity, images from the sections stained with Black-Gold II were converted to grayscale in ImageJ and automated measurements of myelin intensity were taken using the measurement function of ImageJ to record the mean gray value within the regions of interest. All analyses were performed in a blinded fashion.

Statistical analyses. All statistical analyses were performed using the GraphPad Prism software. Statistical significance was determine using unpaired, two-tailed Student's $t$ test or two-way ANOVA with Bonferroni's or Tukey's post hoc multiple- 
bioRxiv preprint doi: https://doi.org/10.1101/2021.05.13.443012; this version posted May 15, 2021. The copyright holder for this preprint (which was not certified by peer review) is the author/funder. All rights reserved. No reuse allowed without permission.

comparison tests. Statistical significance was defined as $p<0.05$. Quantitative data are reported as mean \pm SEM. 


\section{References}

1. Kang, S.H., Fukaya, M., Yang, J.K., Rothstein, J.D. \& Bergles, D.E. NG2+ CNS glial progenitors remain committed to the oligodendrocyte lineage in postnatal life and following neurodegeneration. Neuron 68, 668-681 (2010).

2. Young, K.M. et al. Oligodendrocyte dynamics in the healthy adult CNS: evidence for myelin remodeling. Neuron 77, 873-885 (2013).

3. Chittajallu, R., Aguirre, A. \& Gallo, V. NG2-positive cells in the mouse white and grey matter display distinct physiological properties. J Physiol 561, 109122 (2004).

4. Karadottir, R., Hamilton, N.B., Bakiri, Y. \& Attwell, D. Spiking and nonspiking classes of oligodendrocyte precursor glia in CNS white matter. Nat Neurosci 11, 450-456 (2008).

5. Spitzer, S.O. et al. Oligodendrocyte Progenitor Cells Become Regionally Diverse and Heterogeneous with Age. Neuron 101, 459-471 e455 (2019).

6. Irvine, K.A. \& Blakemore, W.F. A different regional response by mouse oligodendrocyte progenitor cells (OPCs) to high-dose X-irradiation has consequences for repopulating OPC-depleted normal tissue. Eur J Neurosci 25, 417-424 (2007).

7. Hughes, E.G., Kang, S.H., Fukaya, M. \& Bergles, D.E. Oligodendrocyte progenitors balance growth with self-repulsion to achieve homeostasis in the adult brain. Nat Neurosci 16, 668-676 (2013).

8. Robins, S.C. et al. Extensive regenerative plasticity among adult NG2-glia populations is exclusively based on self-renewal. Glia 61, 1735-1747 (2013). 
9. Birey, F. et al. Genetic and Stress-Induced Loss of NG2 Glia Triggers Emergence of Depressive-like Behaviors through Reduced Secretion of FGF2. Neuron 88, 941-956 (2015).

10. Nakano, M. et al. NG2 glial cells regulate neuroimmunological responses to maintain neuronal function and survival. Sci Rep 7, 42041 (2017).

11. Dang, T.C. et al. Powerful Homeostatic Control of Oligodendroglial Lineage by PDGFRalpha in Adult Brain. Cell Rep 27, 1073-1089 e1075 (2019).

12. Nishiyama, A., Komitova, M., Suzuki, R. \& Zhu, X. Polydendrocytes (NG2 cells): multifunctional cells with lineage plasticity. Nat Rev Neurosci 10, 9-22 (2009).

13. Rivers, L.E. et al. PDGFRA/NG2 glia generate myelinating oligodendrocytes and piriform projection neurons in adult mice. Nat Neurosci 11, 1392-1401 (2008).

14. Chang, Y. et al. Ablation of NG2 proteoglycan leads to deficits in brown fat function and to adult onset obesity. PLoS One 7, e30637 (2012).

15. Ziskin, J.L., Nishiyama, A., Rubio, M., Fukaya, M. \& Bergles, D.E. Vesicular release of glutamate from unmyelinated axons in white matter. Nat Neurosci 10, 321-330 (2007).

16. Marques, S. et al. Oligodendrocyte heterogeneity in the mouse juvenile and adult central nervous system. Science 352, 1326-1329 (2016).

17. Vanlandewijck, M. et al. A molecular atlas of cell types and zonation in the brain vasculature. Nature 554, 475-480 (2018).

18. Zhu, L. et al. NG2 expression in microglial cells affects the expression of neurotrophic and proinflammatory factors by regulating FAK phosphorylation. Sci Rep 6, 27983 (2016). 
19. Schatteman, G.C., Morrison-Graham, K., van Koppen, A., Weston, J.A. \& Bowen-Pope, D.F. Regulation and role of PDGF receptor alpha-subunit expression during embryogenesis. Development 115, 123-131 (1992).

20. Pringle, N.P., Mudhar, H.S., Collarini, E.J. \& Richardson, W.D. PDGF receptors in the rat CNS: during late neurogenesis, PDGF alpha-receptor expression appears to be restricted to glial cells of the oligodendrocyte lineage. Development 115, 535-551 (1992).

21. Kessaris, N. et al. Competing waves of oligodendrocytes in the forebrain and postnatal elimination of an embryonic lineage. Nat Neurosci 9, 173-179 (2006).

22. $\mathrm{Xu}, \mathrm{X}$. et al. Selective expression of $\mathrm{Nkx}-2.2$ transcription factor in chicken oligodendrocyte progenitors and implications for the embryonic origin of oligodendrocytes. Mol Cell Neurosci 16, 740-753 (2000).

23. Lu, Q.R. et al. Sonic hedgehog--regulated oligodendrocyte lineage genes encoding bHLH proteins in the mammalian central nervous system. Neuron 25, 317-329 (2000).

24. Tsai, H.H., Macklin, W.B. \& Miller, R.H. Distinct modes of migration position oligodendrocyte precursors for localized cell division in the developing spinal cord. J Neurosci Res 87, 3320-3330 (2009).

25. Doetsch, F., Garcia-Verdugo, J.M. \& Alvarez-Buylla, A. Regeneration of a germinal layer in the adult mammalian brain. Proc Natl Acad Sci U S A 96, 11619-11624 (1999).

26. Menn, B. et al. Origin of oligodendrocytes in the subventricular zone of the adult brain. J Neurosci 26, 7907-7918 (2006). 
27. Xing, Y.L. et al. Adult neural precursor cells from the subventricular zone contribute significantly to oligodendrocyte regeneration and remyelination. $J$ Neurosci 34, 14128-14146 (2014).

28. Brousse, B., Magalon, K., Durbec, P. \& Cayre, M. Region and dynamic specificities of adult neural stem cells and oligodendrocyte precursors in myelin regeneration in the mouse brain. Biol Open 4, 980-992 (2015).

29. Samanta, J. et al. Inhibition of Gli1 mobilizes endogenous neural stem cells for remyelination. Nature 526, 448-452 (2015).

30. Zilkha-Falb, R., Kaushansky, N. \& Ben-Nun, A. The Median Eminence, A New Oligodendrogenic Niche in the Adult Mouse Brain. Stem Cell Reports 14, 1076-1092 (2020).

31. Lin, R. \& Iacovitti, L. Classic and novel stem cell niches in brain homeostasis and repair. Brain Res 1628, 327-342 (2015). 

bioRxiv preprint doi: https://doi.org/10.1101/2021.05.13.443012; this version posted May 15, 2021. The copyright holder for this $p$
Exon I
was hot ldertified by peer review) is the author/funder. All rights reserved. No reuse allowed without permission. Animals sacrificed on specific
day post pump removal

Pdgfra : DTA

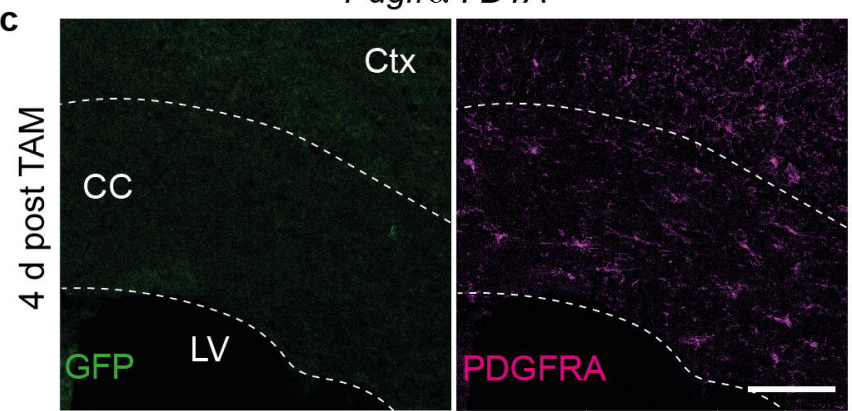

Pdgfra : DTA

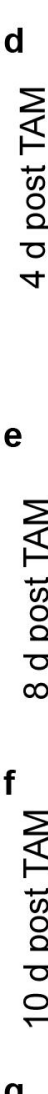

g

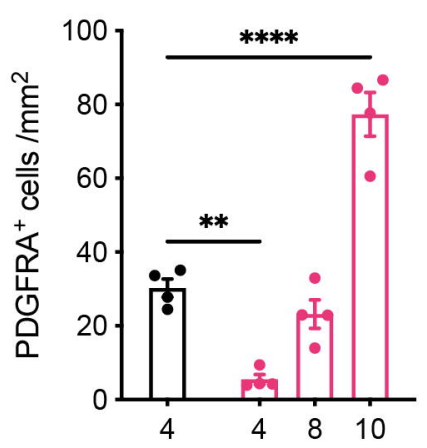

Days after TAM
Pdgfra ${ }^{+}:$DTA $^{+}$, TAM + vehicle

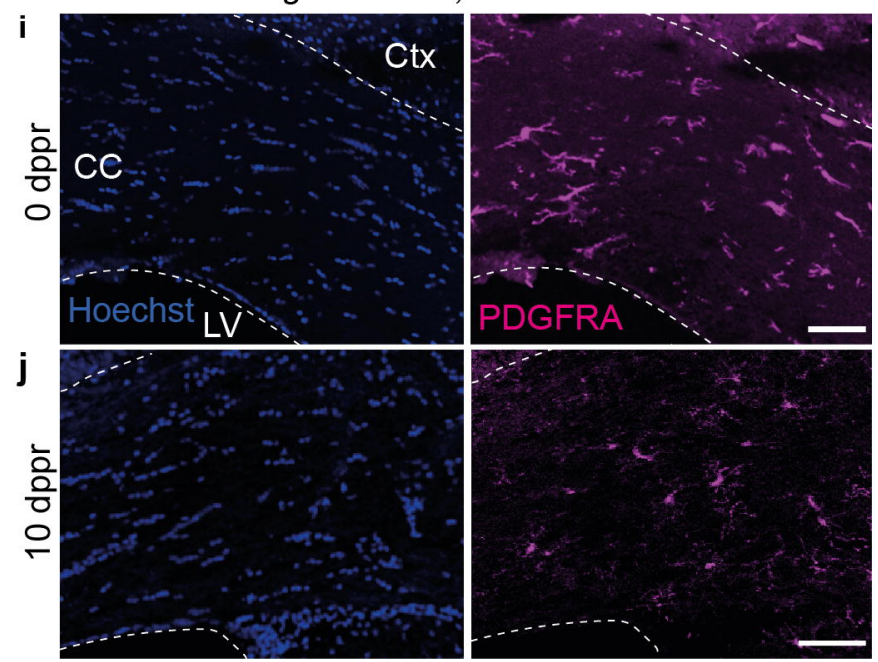

Pdgfra ${ }^{+}:$DTA $^{+}, \mathrm{TAM}+\mathrm{AraC}$
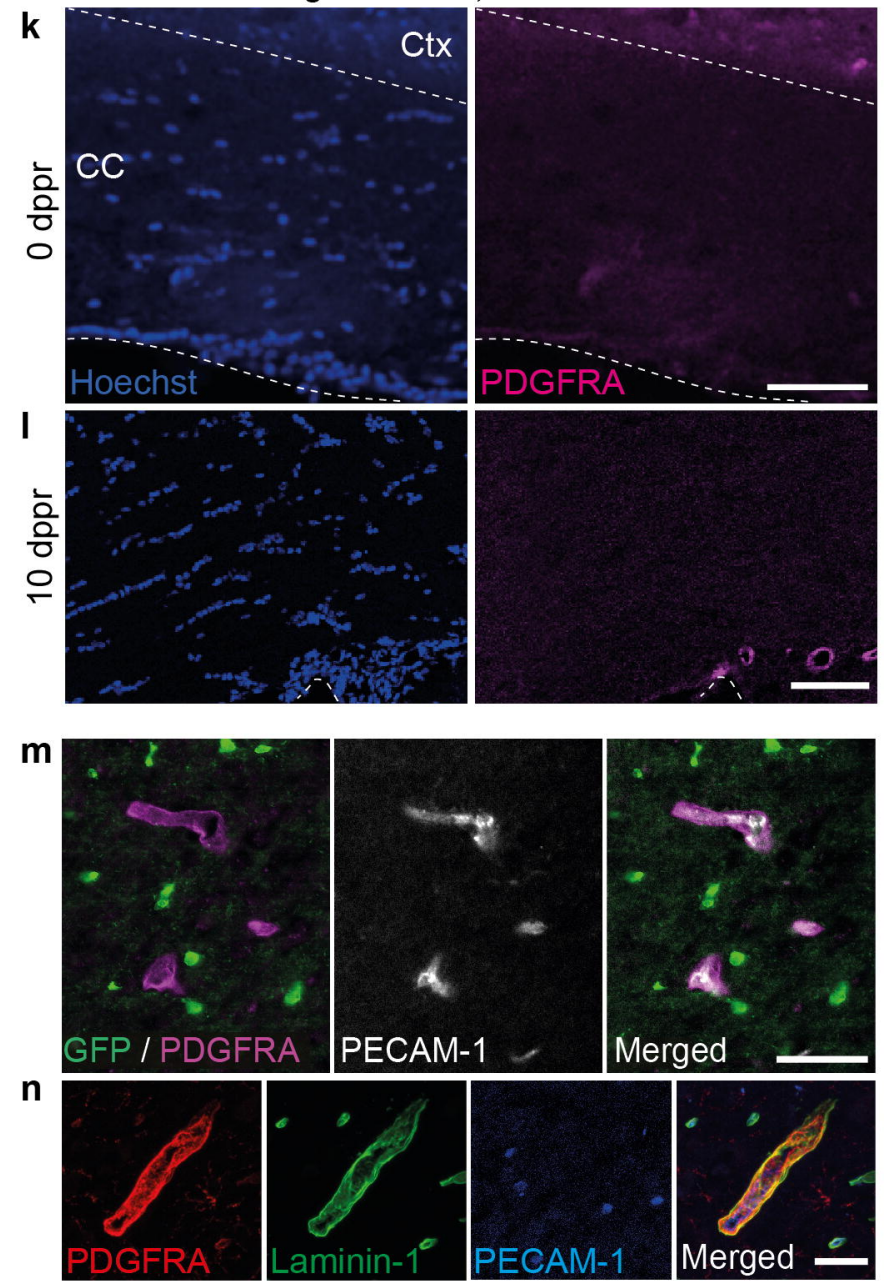

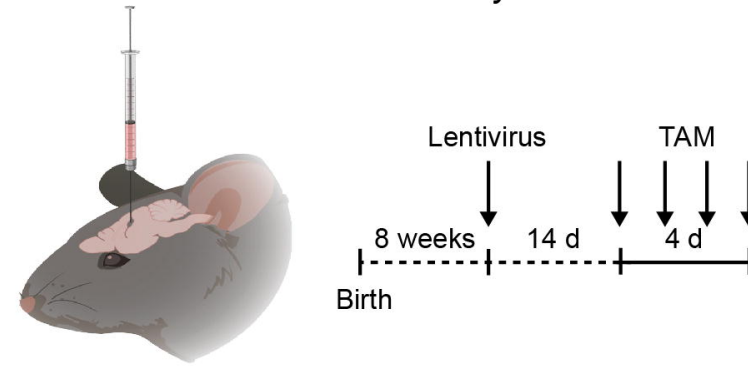

Birth

$4 d$
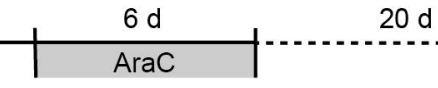

Sacrificed

b

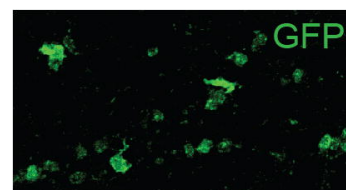

C
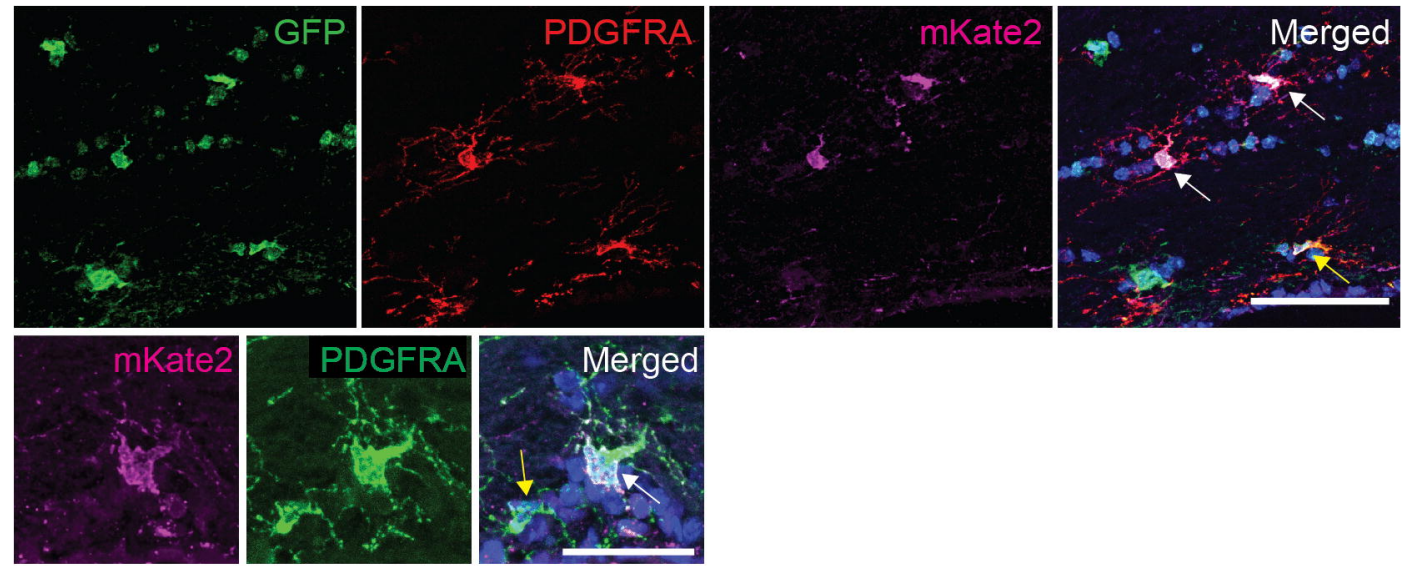

Figure 5 
$20 \mathrm{dppr}$
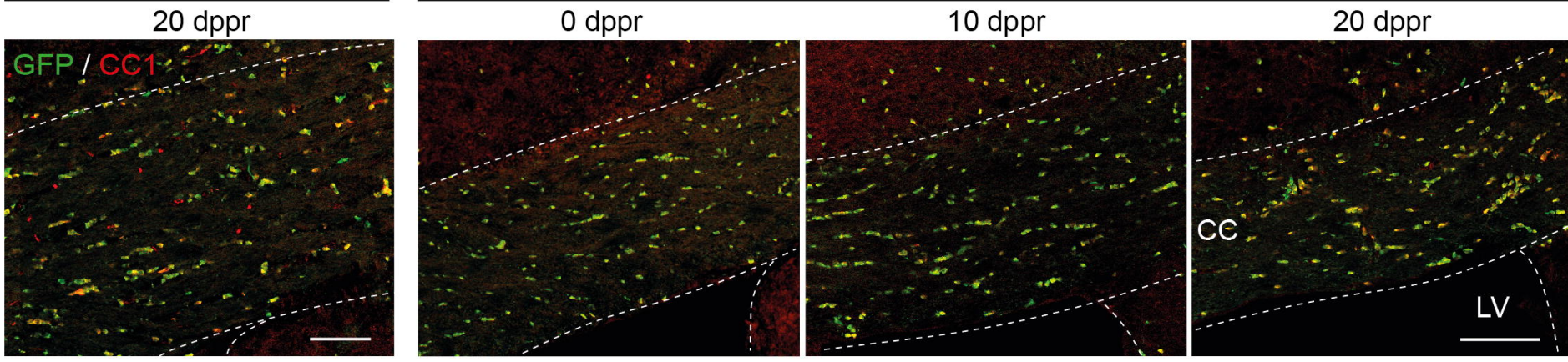

C
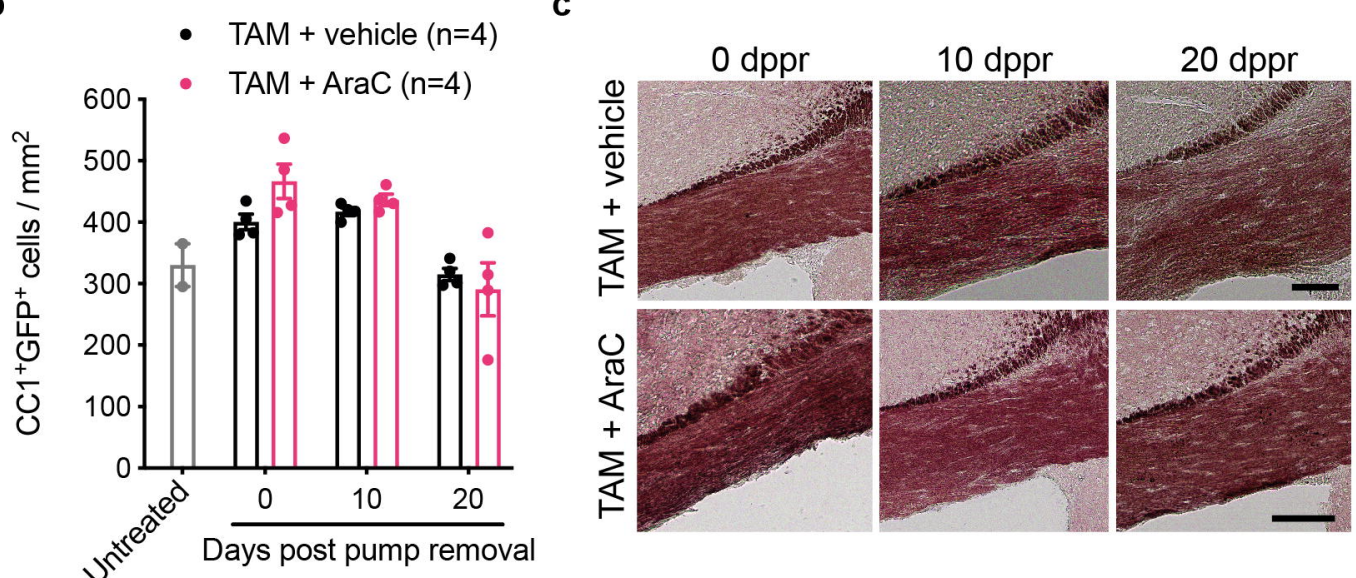

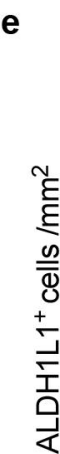

- $T A M+$ vehicle $(n=4)$

- $T A M+\operatorname{AraC}(n=4)$

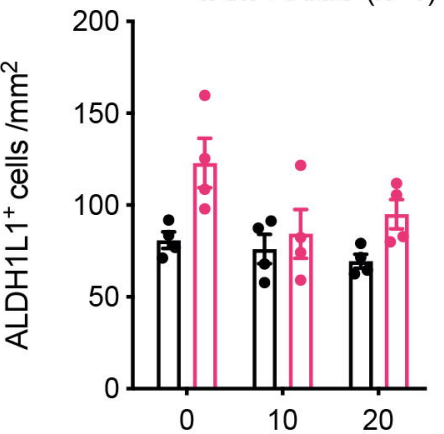

Days post pump removal

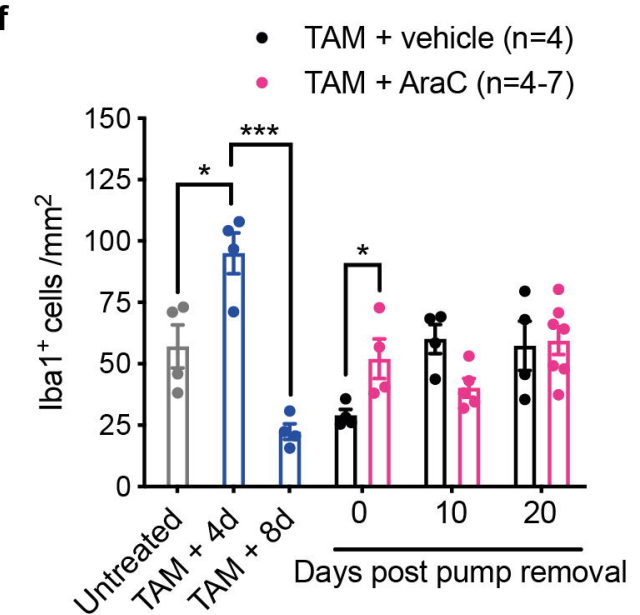

g

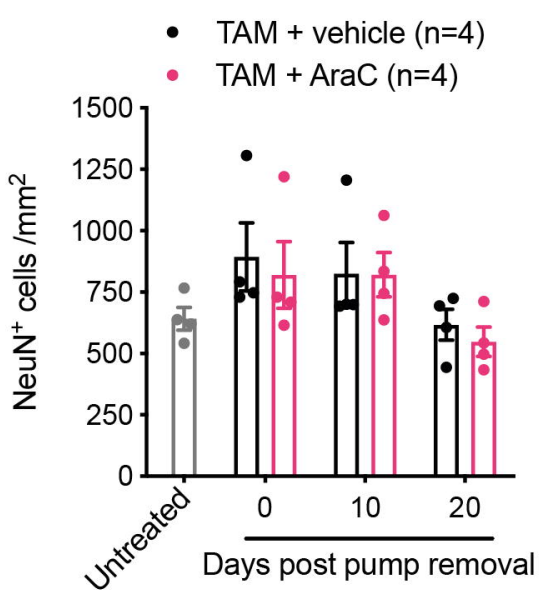

